\title{
Vers de nouveaux récits médiatiques sur l'école au Royaume-Uni ?
}

Media and education in UK

¿Hacia nuevos relatos mediáticos sobre la escuela en el Reino Unido?

Peter Wilby

Traducteur : Robert Elbaz

\section{(2) OpenEdition}

Journals

Édition électronique

URL : http://journals.openedition.org/ries/3990

DOI : 10.4000/ries.3990

ISSN : 2261-4265

Éditeur

Centre international d'études pédagogiques

\section{Édition imprimée}

Date de publication : 1 septembre 2014

Pagination : 31-42

ISBN : 978-2-85420-604-3

ISSN : $1254-4590$

Référence électronique

Peter Wilby, "Vers de nouveaux récits médiatiques sur l'école au Royaume-Uni ? », Revue internationale d'éducation de Sèvres [En ligne], 66 I septembre 2014, mis en ligne le 01 septembre 2016, consulté le 22 juin 2020. URL : http://journals.openedition.org/ries/3990 ; DOI : https://doi.org/10.4000/ries.3990 


\section{Vers de nouveaux récits médiatiques sur l'école au Royaume-Uni ?*}

\section{Peter Wilby}

Aujourd'hui, pratiquement tous les journaux britanniques nationaux disposent d'un correspondant en charge de l'éducation, couvrant l'actualité des écoles, des établissements d'enseignement supérieur et des universités. Si leur nombre est plus réduit qu'il y a vingt ans, lorsque certains journaux employaient jusqu'à trois spécialistes, cela reflète davantage les difficultés financières sans cesse croissantes des journaux ainsi que la charge de travail plus importante des journalistes qu'une réduction quelconque de la couverture des problèmes éducatifs. Certains journaux publient des rubriques hebdomadaires consacrées à l'éducation, quoique celles-ci soient également plus succinctes qu'il y a dix ans. Par exemple, la rubrique éducative du Guardian, publiée le mardi et intitulée Education Guardian, était à une époque un supplément séparé de douze pages, voire davantage. Ce supplément a disparu, remplacé par une rubrique moitié moins longue incluse dans le journal lui-même.

\section{L'ÉDUCATION : UNE PRÉOCCUPATION CROISSANTE}

L'éducation demeure cependant un sujet bien plus débattu qu'il ne l'était il y a cinquante ans. Avant les années 1960, il était à peine considéré comme un sujet d'actualité. Même si les conférences annuelles des syndicats d'enseignants attiraient l'attention, y compris immédiatement après la Seconde Guerre mondiale, principalement parce qu'elles se tenaient (et se tiennent encore) à Easter, ville où les nouvelles saillantes sont rares, la presse "populaire » avait tendance à se concentrer presque exclusivement sur les salaires des enseignants, les associant fréquemment aux difficultés de recrutement (Cunningham, 1992). Une couverture plus exhaustive, particulièrement dans la presse dite " de qualité ", mettait plutôt l'accent sur les universités (et tout spécialement Oxford et Cambridge), fréquentées alors par moins de $6 \%$ des jeunes britanniques, et sur les écoles privées et payantes (telles que Eaton, Harrow et Rugby) plutôt que sur les établissements financés par les deniers publics et fréquentés par la majorité de la population.

\footnotetext{
* Article traduit par Robert Elbaz.
} 
Selon une estimation, il n'y avait au début des années 1960 que trois journalistes à plein temps chargés de l'éducation, à l'exclusion de ceux travaillant pour les publications spécialisées. Ce n'est qu'en 1962 qu'un groupe de correspondants éducatifs fut mis en place afin de coordonner l'accès des journalistes aux ministres et aux autres sources clefs de l'information, alors qu'un groupe de correspondants chargés du travail et de l'industrie fut créé dès 1937, et qu'une association de journalistes chargés des affaires criminelles le fut en 1945 (Williams, 2009). Au début des années 1970, le groupe des correspondants éducatifs était fort d'environ cinquante membres. Dans le Times, le nombre d'articles portant sur l'éducation est passé de 70 en 1960 à 184 en 1967 (Kogan, 1975). À l'époque actuelle, le moteur de recherche du site Web du Times recense 439 articles consacrés à l'éducation en 2013. Au milieu des années 2000, des entretiens avec des journalistes éducatifs ont montré que la plupart d'entre eux considéraient l'éducation comme "l'un des domaines les plus hautement spécialisés du reportage, (...) comparable en importance (...) à la santé, aux affaires judiciaires et au commerce ou à la finance ». Bien que peu d'entre eux puissent remonter suffisamment loin dans le passé pour en être certains, le consensus général était qu'il se situait hiérarchiquement bien plus haut qu'auparavant (Hargreaves et al., 2007).

Les facteurs principaux permettant d'expliquer cette montée en puissance de la couverture de l'éducation, tout autant à la radio et la télévision que dans la presse écrite, ont été la politisation et la centralisation de l'éducation.

\section{LE RÔLE MOTEUR DE LA POLITISATION}

La politisation a débuté à la fin des années 1950 et durant les années 1960, époque à laquelle la Grande-Bretagne a commencé à supprimer la plupart de ses " grammar schools ${ }^{1}$ ", qui sélectionnaient les enfants les plus aptes au plan scolaire après un concours passé à 11 ans, et à les remplacer par des "comprehensive schools ${ }^{2}$ » couvrant l'ensemble des capacités et des compétences. Bien que les différences séparant les principaux partis en matière de sélection scolaire n'aient jamais été rigides (les conseils municipaux dirigés par les Conservateurs ont été parmi les pionniers des comprehensive schools et, jusqu'à aujourd'hui, la politique officielle des conservateurs soutient ce type d'établissements), le parti travailliste a toujours été plus désireux de mettre un terme définitif aux grammar schools, au besoin par décret gouvernemental.

Jusqu'à la fin des années 1970, cependant, l'opinion publique dans son ensemble acceptait que le contenu et les méthodes de l'enseignement fussent du ressort exclusif des enseignants. Comme le déclarait en 1960 un ministre de l'éducation, le curriculum était le « jardin secret » des enseignants, précisant que

1. Établissements traditionnels ne comportant que des sections dites classiques (lettres et sciences). (NdT)

2. Etablissements polyvalents, regroupant des sections classiques et des sections techniques. (NdT) 
« le Parlement ne tenterait jamais d'imposer le curriculum». À Westminster ${ }^{3}$ et à Whitehall ${ }^{4}$, le sujet ne souffrait aucune contestation. Un "parlement » de l'éducation chargé du curriculum et des examens, le School Council ${ }^{5}$, a été mis en place en 1965 mais il ne disposait d'aucun pouvoir de législation ou de définition des objectifs. C'était essentiellement un atelier de parole dans lequel les syndicats d'enseignants et les associations de professeurs enseignant la même matière étaient majoritaires, bien que les gouvernements locaux et national y aient été représentés. En dehors de la classe, la quasi-totalité du pouvoir en matière éducative était entre les mains des conseils municipaux. Bien que la plus grande partie de leur financement vînt du gouvernement central, ils contrôlaient la distribution de l'argent et décidaient quels élèves devaient aller dans quelles écoles. David Eccles, ministre de l'éducation de 1954 à 1957, s'est plaint de ce que, «étant parvenu à obtenir le soutien du cabinet afin d'augmenter le financement de l'éducation, il n'avait pas son mot à dire dans la façon dont ces fonds devraient être dépensés» (Jarvis, 2014).

Ainsi, l'éducation était couverte de manière plus exhaustive et, mieux encore, traitée avec plus de considération dans les journaux locaux qu'elle ne l'était dans les journaux nationaux britanniques ou à la radio et à la télévision. La presse nationale, habituée à recevoir la plupart de ses consignes de Whitehall, de Westminster et des corps constitués associés au gouvernement central, éprouvait quelques difficultés à dégager des informations significatives sur le sujet. L'opinion dominante était qu'une querelle portant sur les admissions scolaires à Newcastle n'intéresserait pas les lecteurs de Birmingham. De même, une innovation dans le curriculum à Birmingham ne serait d'aucun intérêt pour les lecteurs de Newcastle. Ce n'est que dans les années 1960 que le nombre croissant de journalistes spécialistes de l'éducation commença à faire émerger des développements locaux dignes d'intégrer la presse nationale. Par exemple, les comprehensive schools fraîchement ouvertes pouvaient bénéficier d'une couverture nationale, tout comme les changements radicaux opérés dans l'organisation interne des établissements, le curriculum et les méthodes pédagogiques, qui furent rangés de manière assez large sous l'intitulé "progressiste ». Mais les universités et les pensionnats privés et payants (connus en Angleterre sous l'appellation trompeuse de "public schools") ont continué à bénéficier de davantage de couverture médiatique, parce qu'ils attiraient une clientèle nationale constituée des classes moyennes supérieures, le segment le plus recherché par les publicitaires, même dans les journaux les plus ordinaires.

À cette époque, presque tous les journalistes éducatifs soutenaient les comprehensive schools et les approches pédagogiques "progressistes ». En 1972, un éditorialiste de l'hebdomadaire de droite The Spectator se plaignait que, à

\footnotetext{
3. Siège du Parlement britannique. (NdT)

4. Quartier du centre de Londres où se trouvent la plupart des grandes administrations et des ministères. (NdT)

5. Conseil des écoles. ( $N d T)$
} 
l'exception d'un seul, les 48 membres du groupe des correspondants éducatifs étaient "de gauche, et certains d'extrême-gauche ». Même dans les journaux conservateurs, ils pouvaient mettre en avant la cause "progressiste », ou du moins lui préparer le terrain, principalement parce que les rédacteurs en chef ne montraient que fort peu d'intérêt pour l'éducation. C.B. Cox, un professeur d'anglais de l'université de Manchester, a déclaré en 1981, lors d'une conférence en Australie, que les journaux avaient joué un rôle prépondérant pour convaincre le public des mérites de la pédagogie "progressiste ». À la fin des années 1960 et au début des années 1970, Cox, ainsi que des collègues partageant la même approche, ont publié une série de Black Papers ${ }^{6}$, rédigés par des chercheurs et des enseignants partisans d'une pédagogie " traditionnelle », qui ont délibérément tenté de renverser le courant ou, comme le dit Cox, de "déplacer le centre de gravité » du débat. Ces articles étaient rédigés dans un style accessible, populiste, dépourvu de tout jargon, et ont fait l'objet d'une intense promotion auprès des journaux. Les rédacteurs des Black Papers comprenaient qu'ils pouvaient maximiser la couverture médiatique favorable en expédiant le pamphlet aux journaux le dimanche, jour où l'information était rare et où la plupart des journalistes spécialisés ne travaillaient pas, afin qu'ils puissent le publier le lundi matin. Un ministre travailliste de l'éducation leur a étourdiment fait une publicité involontaire en faisant référence à leur premier article comme étant «le jour le plus sombre du système éducatif britannique depuis plus d'un siècle ».

Les Black Papers et leur descendance, telle que le National Council for Educational Standards (Conseil national pour les standards éducatifs) qui, là encore pour promouvoir la couverture médiatique, tenait ses conférences le dimanche, inversèrent effectivement la tendance. Les "traditionnalistes» n'hésitaient pas à courtiser les journalistes, à faire en sorte que la presse ait libre accès à leurs dirigeants et à essayer de capter l'attention du plus grand nombre. Par contraste, les "progressistes » considéraient le populisme avec dédain et la presse «capitaliste » avec méfiance. Par exemple, on ne pouvait joindre la porteparole de la campagne pour la promotion de l'éducation globale qu'avec les pires difficultés. De plus, elle ne faisait aucun effort pour cacher son hostilité envers les journalistes et ne les autorisait pas à la citer.

Malgré tous les efforts des rédacteurs des Black Papers et de leurs partisans auprès des médias, la montée en puissance des comprehensive schools se poursuivit au début sans interruption: dans les années 1970, bien que Margaret Thatcher en personne fût ministre de l'éducation dans la première moitié de la décennie, il en fut créé davantage que jamais auparavant. Les grammar schools ne survécurent que dans une poignée de zones sous contrôle de conseils conservateurs purs et durs. Les médias n'ont que peu de pouvoir en matière éducative (et en fait plus généralement en politique), du moins dans le

6. Série d’articles équivalant à un « Livre noir ». $(N d T)$ 
sens de "capacité de transformation » telle qu'elle est définie par le sociologue Anthony Giddens : "la capacité d'intervenir sur des événements de manière à en modifier le cours ». Mais ils créent toutefois un ordre du jour et un cadre de travail - parfois extrêmement restrictifs - pour le débat. Dans les années 1970, les médias, influencés par les Black Papers, commencèrent lentement à modifier cet ordre du jour. Les journaux se faisaient volontiers l'écho des recherches soulignant les faiblesses des méthodes pédagogiques "progressistes » et montèrent en épingle le cas d'une école primaire londonienne qui, de toute évidence, était allée trop loin dans lesdites méthodes et dont les enseignants entraînaient apparemment les élèves à devenir les membres d'une avant-garde révolutionnaire, tout en négligeant totalement les fondamentaux de la lecture et du calcul.

L'intérêt du public pour l'éducation ne cessait de croître, et pas seulement parce que le débat médiatique était de plus en plus vigoureux et polarisé. Durant la plus grande partie du $\mathrm{XX}^{\mathrm{e}}$ siècle, l'éducation n'a joué qu'un rôle mineur dans la vie de la majorité de la population. La plupart des gens quittaient l'école vers 15 ans pour ne jamais retrouver une éducation à plein temps. Ils ne passaient aucun examen et n'avaient aucun diplôme. Quelques-uns pouvaient éventuellement retourner à l'université un jour ou deux par semaine afin d'acquérir les compétences nécessaires à l'exercice d'un métier, mais beaucoup se formaient sur le tas ou trouvaient des emplois de manœuvres ou d'ouvriers non qualifiés, alors courants. Même de nombreux emplois de bureau ne nécessitaient que des qualifications minimales. Le journalisme lui-même n'exigeait rien de plus que des connaissances rudimentaires de sténographie et de droit.

Durant la seconde moitié du $\mathrm{XX}^{\mathrm{e}}$ siècle, cependant, les perspectives de carrière - et, alors que les industries lourdes battaient de l'aile, les chances mêmes de trouver un emploi - dépendaient de plus en plus de la longueur des études et, particulièrement, des qualifications acquises à l'école. L'éducation devint la détentrice des opportunités de toute une vie et, par là-même, une source d'inquiétude croissante de la part des parents de toutes conditions. Cela contribua à en faire un sujet d'importance, non seulement pour les journaux et leurs lecteurs, mais aussi pour les politiques.

\section{L'AUTRE MOTEUR : LA CENTRALISATION}

La gouvernance décentralisée du système scolaire anglais avait longtemps été une source de frustration, à la fois pour les politiques de Westminster et les fonctionnaires de Whitehall. Les ministres de l'éducation fourmillaient souvent d'idées sur la façon d'améliorer l'école mais ils découvraient qu'ils ne disposaient d'aucun levier pour les faire aboutir. Par exemple, lorsque Sir Keith Joseph, l'un des principaux architectes de ce qui devait devenir le thatchérisme, devint ministre de l'éducation en 1981, il voulut que les établissements suscitassent une attitude plus favorable au monde de l'entreprise et au commerce parmi les jeunes. 
À son grand désespoir, il se trouva dans l'incapacité de mettre en œuvre ce changement. Comme tous ses prédécesseurs, il n'était rien de plus qu'un simple spectateur, alors même que les syndicats d'enseignants et les autorités éducatives locales (qui reposaient sur les conseils municipaux) - au sein desquelles les journalistes spécialisés cultivaient des contacts de manière aussi assidue qu'ils le faisaient parmi les ministres, leurs conseillers et les fonctionnaires - tiraient les ficelles.

En grande partie parce qu'il avait très peu de pouvoir direct, Joseph avait absolument besoin de l'appui du public pour exercer une pression sur les décisionnaires et faire triompher ses idées. Par exemple, il souhaitait changer les pratiques de travail des enseignants et introduire une évaluation régulière de leurs performances. À l'époque, cela s'appelait "évaluation de l'enseignant ${ }^{7}$ ». Mais, parce qu'il ne les employait pas lui-même, pas plus qu'il ne déterminait combien et de quelle manière ils étaient payés - bien qu'il finançât en effet les augmentations des personnels -, il devait absolument exercer une pression sur les employés des conseils municipaux et sur les syndicats d'enseignants qui négociaient les conditions de salaire et de travail par l'intermédiaire d'une machinerie quelque peu complexe connue sous le nom de "comité Burnham ». Le soutien du public et, par là-même celui de la presse, était essentiel pour mettre en œuvre ses idées.

Il a en grande partie échoué. Les syndicats enseignants mirent sur pied une campagne extrêmement efficace soulignant les faibles salaires des enseignants, qui obligeaient certains à avoir un second métier, leur lourde charge de travail ainsi que leur propension aux infarctus et aux dépressions nerveuses. Par contraste, Joseph était tout à la fois « incompétent et indifférent » pour influencer les médias, au grand désespoir des attachés de presse du ministère. "Il était très souvent réticent à l'idée de rencontrer les journalistes, et menait ses conférences de presse avec un manque d'entrain à peine caché et à une allure indécente. » (Wilby, 1986).

Ce n'est que lorsque Joseph quitta son poste en 1986 que le Education Reform $A c t^{8}$ de Kenneth Baker commença à faire pencher la balance du pouvoir. Comme pratiquement tous les successeurs de Joseph (à une exception notable près), Baker maîtrisait davantage les médias. Il a inauguré le premier Curriculum national, la première loi permettant aux écoles de se libérer de la tutelle des conseils municipaux, et les premières expériences menées afin de persuader les sponsors privés de financer le système scolaire d'État. Il a également résolu le problème auquel Joseph avait été confronté dans sa tentative d'influencer les conditions de salaire et de travail des enseignants, en abolissant la machinerie du comité Burnham et en se donnant les moyens de fixer les contrats de travail des enseignants. 
À partir du milieu des années 1980, les gouvernements conservateurs de Margaret Thatcher furent à l'origine de changements spectaculaires en Angleterre et au pays de Galles (l'Écosse et l'Irlande du Nord ont des systèmes éducatifs séparés, sujets à des régimes séparés) qui furent largement entérinés par les gouvernements travaillistes de Tony Blair à partir de 1997, et développés par la coalition libérale-démocrate-conservatrice à partir de 2010. On donna aux parents plus de latitude dans le choix des établissements et plus de poids dans la façon dont ceux-ci fonctionnaient. On obligea les établissements à publier leurs résultats d'examens sous une forme standardisée. Les conseils municipaux furent sommés de les financer selon une formule définie à l'échelle nationale et de déléguer les décisions relatives aux dépenses aux écoles, laissant le soin aux membres des conseils d'établissement, ainsi qu'aux directeurs, principaux et proviseurs, de déterminer, par exemple, les sommes à consacrer à l'achat de manuels ou aux réparations.

On donna aux établissements l'opportunité cruciale de s'affranchir du contrôle des conseils municipaux et de devenir semi-indépendants, recevant leurs financements directement du gouvernement central. Une majorité d'établissements secondaires a désormais opté pour cette solution. Des compagnies privées et des associations furent conviées à mettre en place de nouvelles écoles et à prendre le contrôle de celles qui existaient déjà. Par intermédiaire d'une nouvelle instance de régulation, l'OFSTED (Office for Standards in Education ${ }^{9}$ ), les pouvoirs des inspecteurs de sa Majesté furent grandement renforcés et les ministres de l'éducation prirent de plus en plus l'initiative de changements de direction d'établissement ou même de fermetures, dans le cas d'établissements " défaillants ». Évolution décisive, on demanda aux établissements de se conformer à un Curriculum national que différents ministres déterminèrent, de façon plus ou moins détaillée. Ayant pour la plupart suivi des études classiques, les ministres étaient particulièrement soucieux de spécifier quels romans, quelles pièces et quels poèmes devraient être étudiés en littérature anglaise et quels événements devaient l'être en histoire. Le parti travailliste n'a pas seulement édicté le contenu du curriculum mais il a également défini quelles méthodes devaient être utilisées pour enseigner la lecture et le calcul dans les écoles primaires.

Tout cela conduisit à une explosion de l'intérêt des médias. Le jardin secret était grand ouvert, permettant à tout un chacun d'en piétiner les platesbandes. Dans le passé, la simple et spectaculaire confrontation des contraires dont sont friands les médias n'était déclenchée que par les querelles relatives aux salaires des enseignants. Celles-ci n'étaient connectées aux problèmes pédagogiques que de façon périphérique. Elles étaient couvertes comme de simples luttes entre employeurs et employés, semblables à n'importe quel autre conflit du travail. Voilà que ces conflits portaient maintenant sur quoi et comment enseigner, générant des débats hautement politisés, portant par exemple sur la façon dont

9. Bureau des référentiels éducatifs, des services à l'enfant et des compétences. (NdT) 
on devrait enseigner l'histoire de l'Empire britannique ou sur le fait de savoir si les cours d'anglais devaient se concentrer plutôt sur la création libre ou sur la correction de la grammaire et de l'orthographe.

\section{CADRER Le dÉBAT : LE RÉCIT OPPOSANT «TRADITIONALISTES " ET «PROGRESSISTES »}

Les médias nationaux réduisaient ces conflits à une simple opposition entre les "traditionnalistes », principalement de droite, et les "progressistes », principalement de gauche. Les premiers mettaient particulièrement l'accent sur la littéracie et la numéracie fondamentales, sur des matières comme la physique, la chimie, l'histoire et la littérature, sur une instruction formelle dispensée à des élèves assis en rang à des bureaux face au tableau alors que leur enseignant leur explique quoi apprendre, sur une sélection vers différentes " filières » au sein des établissements et, si possible, entre établissements, sélection assurée par les capacités des élèves, sur des examens écrits à l'ancienne, durant généralement trois heures. Les derniers défendaient un curriculum plus flexible ne divisant pas le champ de la connaissance en frontières rigides entre matières, un apprentissage informel par la découverte, où les élèves sont assis en groupes et sont encouragés par leurs enseignants à découvrir les choses par eux-mêmes, les comprehensive schools comportant des classes hétérogènes, les examens d'un nouveau genre, comprenant une évaluation en contrôle continu et des projets menés à bien dans le laps de temps choisi par les élèves. La plupart des enseignants sont des pragmatiques qui n'ont choisi aucun des deux camps. Ils préfèrent un curriculum équilibré et un panachage de méthodes adaptées à la pédagogie comme à l'évaluation. Mais les médias préféraient subsumer chaque problème pédagogique ou presque dans le cadre du conflit entre progressistes et traditionnalistes. Par exemple, on pensait souhaitable de donner aux parents un choix plus grand d'écoles et au secteur privé plus de liberté de les diriger parce que ces deux options ramèneraient inévitablement les écoles aux fondamentaux. Les politiques, occupés à défendre leurs propres intérêts, ont encouragé sans aucun état d'âme les journalistes et les commentateurs à rendre compte de l'ensemble des problèmes éducatifs à la lumière de cette dichotomie.

À la fin des années 1980, le discours dominant des articles des médias était presque entièrement consacré à la lutte à mort, du moins le semblait-il, entre «traditionnalistes » et "progressistes " (parfois appelés «trendies ${ }^{10}$ »). Après la politique de centralisation du pouvoir éducatif menée par les conservateurs, tout cela prit une dimension de plus en plus politique. Les événements de 1991 ont montré comment ce discours dominant allait maintenant se développer.

10. Trend signifie « tendance », en anglais. $(N d T)$ 
Cette année-là, le conseil municipal de Leeds publia un rapport sur les résultats d'un programme destiné à transformer ses écoles primaires en étendard des «bonnes pratiques primaires », ce qui signifiait - où était censé signifier - les rendre plus " progressistes ». Normalement, un tel rapport provenant d'une autorité pédagogique de province ne devrait attirer l'attention de la presse nationale que fort peu. Dans ce cas précis, personne ne sait pourquoi les choses furent différentes. Peut-être cela tint-il à la date de publication, fin juillet, lorsque les journalistes n'ont rien à se mettre sous la dent. Mais quelques commentateurs suggérèrent que le gouvernement conservateur, confronté à une élection générale périlleuse l'année suivante, avait renseigné certains des correspondants spécialisés basés à Londres, y voyant une occasion de discréditer un conseil municipal contrôlé par le parti travailliste et d'associer ainsi leurs principaux adversaires politiques à un échec pédagogique patent.

Quelle qu'en soit la raison, la couverture du reportage écrit par Robin Alexander, professeur d'enseignement primaire à l'université de Leeds, porta un coup très sévère à l'éducation dite " progressiste ", probablement le plus sérieux qu'elle ait eu à subir en une décennie. Le Daily Telegraph titra en une "L'enseignement progressiste, un échec de 14 millions de livres sterling ", avant d'ajouter, dans le corps de l'article: "l'éducation de millions d'enfants d'âge primaire a été sabotée au nom d'une idéologie anarchique ». La réaction gouvernementale s'accorda très précisément à cette couverture médiatique. John Major, qui venait de succéder à Margaret Thatcher comme premier ministre conservateur, se servit de ce rapport afin d'encourager " un retour aux fondamentaux » et annonça que « les théoriciens progressistes (avaient) eu leur mot à dire et (...) (avaient) désormais fait leur temps. » Plus tard, en 1992, Alexander fut invité par le gouvernement à participer à une enquête officielle portant sur « les pratiques éducatives à l'école primaire ». Faisaient également partie de cette enquête Jim Rose, alors doyen de l'inspection de l'enseignement primaire, et Chris Woodhead, le directeur du National Curriculum Council, qui acceptaient dans une large mesure la version gouvernementale et médiatique selon laquelle de vaillants traditionnalistes menaient un combat d'arrière-garde contre une puissante machine de guerre progressiste. Nommés juste avant Noël, la presse les surnomma «les trois sages ». Lorsqu'ils publiaient un article, les gros titres des journaux clamaient : «Un appel au retour des cours traditionnels» (Alexander, 1997).

Toutefois, comme l'a montré Alexander, les conclusions principales du rapport de Leeds furent que le projet "était une initiative tout à fait digne de l'investissement de l'Autorité ", même s'il avait mis au jour des faiblesses importantes dans les résultats de certains établissements. Alexander écrivit qu' "une analyse complexe et soigneusement menée (avait) été réduite à une simple pathologie. »Selon lui, les résultats de l'enquête conduite par « les trois sages » furent également déformés, en partie par Woodhead - qui réécrivit ce qui était censé être un avant-projet -, en partie par le communiqué de presse émanant du 
ministère de l'éducation, et en partie enfin par la presse elle-même. Par exemple, le rapport rejetait un retour à la constitution de filières fondées sur les capacités, une requête essentielle des traditionnalistes. Lorsqu'Alexander rendit publiques ses réserves à propos d'un rapport qui avait été publié sous son nom, la presse l'accusa de volte-face et le traita d' "homme peu sage » (Alexander, 1997).

La campagne contre l'enseignement «progressiste » ne se limitait pas aux seuls journaux. La télévision joua également un rôle. À l'automne 1991, dans le sillage du rapport de Leeds, une émission d'actualité prestigieuse de la BBC rapporta que « des experts souffrant de la maladie de l'éducation progressiste (avaient) répandu un chancre dans les salles de classe britanniques ». Pour illustrer cette thèse, l'émission se rendit dans deux écoles primaires où, selon une étude, " on filma un échantillon de pratiques pédagogiques très bref et non représentatif, réduit à sa plus simple expression après la mise en forme et le montage. Placé hors de son contexte, le matériau qui passa à l'antenne fut utilisé pour décrire l'approche progressiste en termes simplistes» (Wallace, 1993).

Le contenu des articles ne changea que très peu au cours des vingt années qui suivirent. Comme la «menace rouge » de l'époque de la guerre froide, les «progressistes » étaient toujours prêts à quelque mauvais coup et leur mission ne connaissait aucun répit afin de subvertir les valeurs traditionnelles, raisonnables et décentes. Cette approche était rendue plus plausible encore par le jugement fréquemment répété selon lequel, alors que la droite gagnait les guerres économiques, convertissant le monde entier à la doctrine du marché libre, la gauche, elle, gagnait les guerres culturelles. Selon ce discours dominant, les méthodes pédagogiques à la mode étaient imposées pour des raisons idéologiques par un "establishment pédagogique » comprenant des directeurs et des enseignants en vue, des professeurs de pédagogie, des formateurs, des membres de conseils municipaux, des conseillers et même des inspecteurs pédagogiques de Sa Majesté, dont les avis étaient en leur temps considérés comme le Saint Graal. Même la prise de fonctions d'un gouvernement travailliste en 1997 n'a pas modifié ce discours, bien qu'une partie de sa rhétorique ait été atténuée et que les ministres travaillistes n'aient pas ménagé leurs efforts pour tenir compte de l'opinion des enseignants. La politisation de l'école a connu des extrêmes inimaginables. Même l'enseignement de la lecture, un sujet pourtant éminemment technique, s'est transformé en bataille entre la méthode syllabique, soutenue par la droite, et la méthode globale, soutenue par la gauche.

\section{VERS UNE Ère DE LA COMMUNICATION ?}

Entre-temps, les politiques ont énormément amélioré leur façon d'utiliser les médias à leur profit. Lorsqu'ils définissent une politique, ils ne pensent pas seulement à son efficacité mais surtout à la façon dont ils pourront la «vendre » aux médias. Tous les gouvernements aspirent à garder le contrôle 
de l'agenda médiatique et aucun ministre ne peut survivre suffisamment longtemps s'il ou elle ne peut alimenter les médias par ses "initiatives » politiques. L'un des effets de cette politisation croissante de l'éducation est que les ministres informent les correspondants politiques accrédités à Westminster, et non les spécialistes de l'éducation, de ce qu'ils considèrent comme des "initiatives majeures ». Puisque les journalistes politiques sont par définition des spécialistes de la politique et non de l'éducation (ou de la santé, du maintien de l'ordre ou des services sociaux), les politiques conduites en matière éducative sont rarement examinées de façon critique. De plus, le nombre croissant d'éditorialistes (pratiquement chaque journal publie deux ou trois éditoriaux chaque jour), qui expriment des opinions sur un éventail très large de problèmes et sont employés plus pour leur faculté d'écrire de façon provocante et amusante que pour leurs compétences sur un sujet particulier, marginalise un peu plus encore leur rôle d'experts et d'enquêteurs bien informés. Les professeurs de pédagogie et autres spécialistes universitaires sont presque totalement exclus à la fois des décisions politiques et de la couverture médiatique de l'éducation.

La plupart des médias sociaux et des blogs sur Internet multiplient le nombre de commentaires approximatifs. En matière d'éducation, comme c'est le cas dans d'autres domaines, Internet permet à quelques voix bien informées de s'adresser à un large public d'une manière tout à fait nouvelle. Par exemple, le Réseau des écoles locales défend des établissements dirigés par des conseils municipaux élus et critique le nombre croissant d'écoles dirigées par des chaînes privées, appuyé en cela par des commentaires ayant fait l'objet d'une recherche approfondie, publiés sur son site Web.

Mais il s'agit là d'une exception. Le discours mettant en scène les " progressistes » contre les « traditionnalistes » continue de paralyser l'éducation et aucun ministre de l'éducation ne s'en est davantage servi que l'actuel ministre conservateur, Michael Gove. Selon une source, les gouvernements successifs ont mis au point un "objet de dérision » qu'ils jurent ensuite "d'exorciser» (Wallace, 1993). Cela convient parfaitement aux médias, qui disposent ainsi du choc des contraires dont ils raffolent et peuvent faire entrer pratiquement tous les problèmes relatifs à l'éducation dans ce cadre simpliste.

\section{BIBLIOGRAPHIE}

AlEXANDER R. (1997): Policy and Practice in Primary Education, $2^{\mathrm{e}}$ édition, Routledge, Londres.

CUNNINGHAM P. (1992): Teachers' professional image and the press 1950-1990, History of Education: Journal of the History of Education Society, 21: 1, 37-56.

HARGREAVES L., et al. (2007) : The Status of Teachers and the Teaching Profession in England, Department for Education and Skills, Research Report 831B.

JARVIS F. (2014) : You never know your luck: reflections of a cockney campaigner for education, Grosvenor House, Guildford, Surrey. 
KOGAN M. (1975) : Educational Policy-Making: A study of Interest Groups and Parliament, Allen et Unwin, Londres.

WALLACE M. (1993) : "Discourse of derision: the role of the mass media within the education policy process", Journal of Education Policy, 8: 4, 321-337.

WILBY P. (1986) : "Press and Policy: teacher appraisal", Journal of Education Policy, 1: $1,63-72$.

WILLIAMS K. (2009) : Read All About It: A History of the British Newspaper, Routledge, Londres. 